\title{
A Study on Inventory Modeling Through Matrices
}

\author{
C. Velmurugan • R. Uthayakumar
}

Published online: 22 October 2014

(C) Springer India Pvt. Ltd. 2014

\begin{abstract}
An intention of this paper is to study the inventory modelling through matrices and establish the optimum results such as economic order quantity and total inventory cost by using matrix operations. We define various factors which are affecting the inventory cost by using the diagonal matrices. We developed the computation algorithm to test the effectiveness of the model through numerical example.
\end{abstract}

Keywords Inventory $\cdot$ Matrix $\cdot$ Diagonal matrix $\cdot$ Trace of matrix $\cdot$ Ordering diagonal matrix $\cdot$ Total cost diagonal matrix

Mathematics Subject Classification $\quad 90 \mathrm{~B} 05 \cdot 15 \mathrm{~A} 15$

\section{Introduction}

Inventory consists of usable but idle resources which are materials and goods. The amount of material, a company has in stock at a specific time is known as inventory or in terms of money it can be defined as the total capital investment over all the materials stocked in the company at any specific time. Inventory may be in the form of, raw material inventory, in process inventory, finished goods inventory, etc.

As a lot of money is engaged in the inventories along with their high carrying costs, companies cannot afford to have any money tied in excess inventories. Any excessive investment in inventories may prove to be a serious drag on the successful working of an organization. Thus there is a need to manage inventories more effectively to free the excessive amount of capital engaged in the materials.

Inventory control is the supervision of supply, storage and accessibility of items in order to ensure an adequate supply without excessive oversupply. It can also be referred as internal control-an accounting procedure or system designed to promote efficiency or assure the

C. Velmurugan $(\bowtie) \cdot R$. Uthayakumar

Gandhigram Rural Institute-DU, Dindigul, Tamil Nadu, India

e-mail: jsr.maths@gmail.com 
implementation of a policy or safeguards assets or avoid fraud and error, etc. In economics, the inventory control problem, which aims to reduce overhead cost without hurting sales. In the field of loss prevention, systems designed to introduce technical barriers to shoplifting. Also it answers the following basic questions of any supply chain: (1) What to order? (2) When to order? (3) Where to order? (4) How much to carry in stock? so as to gain economics.

In order to control inventories appropriately, one has to consider all cost elements that are associated with the inventories. There are few such cost elements, which do affect cost of inventory. Carrying cost refers to the total cost of holding inventory. This includes warehousing costs such as rent, utilities and salaries, financial costs such as opportunity cost, and inventory costs related to perishability, pilferage, shrinkage and insurance. Ordering cost is cost of ordering a new batch of materials. These include cost of placing a purchase order, costs of inspection of received batches, documentation costs, etc. Economic order quantity (EOQ) is the order quantity of inventory that minimizes the total cost of inventory management. Costs incurred when an item is out of stock; also called stockout costs. These costs include the lost contribution margin on sales plus lost customer. The time gap between placing of an order and its actual arrival in the inventory is known as leadtime. Demand is a buyer's willingness and ability to pay a price for a specific quantity of a good or service. Demand refers to how much (quantity) of a product or service is desired by buyers at various prices. The quantity demanded is the amount of a product people are willing to buy at a certain price; the relationship between price and quantity demanded is known as the demand.

\section{The Basic EOQ Model}

Churchman et al. [1] and Hadley and Whitin [2] the basic EOQ model is a formula for determining the optimal order size that minimizes the sum of carrying costs and ordering costs. The model formula is derived under a set of simplifying and restrictive assumptions, as follows:

- Demand is known with certainty and is constant over time.

- No shortages are allowed.

- The order quantity is received all at once.

The EOQ is the order size that minimizes the sum of carrying costs and ordering costs. These two costs react inversely to each other. As the order size increases, fewer orders are required, causing the ordering cost to decline, whereas the average amount of inventory on hand will increase, resulting in an increase in carrying costs. Thus, in effect, the optimal order quantity represents a compromise between these two inversely related costs.

The total annual ordering cost is computed by multiplying the cost per order, designated as $A$, times the number of orders per year. Since annual demand, $D$, is assumed to be known and to be constant, the number of orders will be $D / Q$, where $Q$ is the order size and

$$
\text { Annual ordering cost }=\frac{A D}{Q} .
$$

The only variable in this equation is $Q$; both $A$ and $D$ are constant parameters. Thus, the relative magnitude of the ordering cost is dependent upon the order size.

Total annual carrying cost is computed by multiplying the annual per-unit carrying cost, designated as $h$, times the average inventory level, determined by dividing the order size, $Q$, by $2: Q / 2$;

$$
\text { Annual carrying cost }=\frac{h Q}{2} .
$$




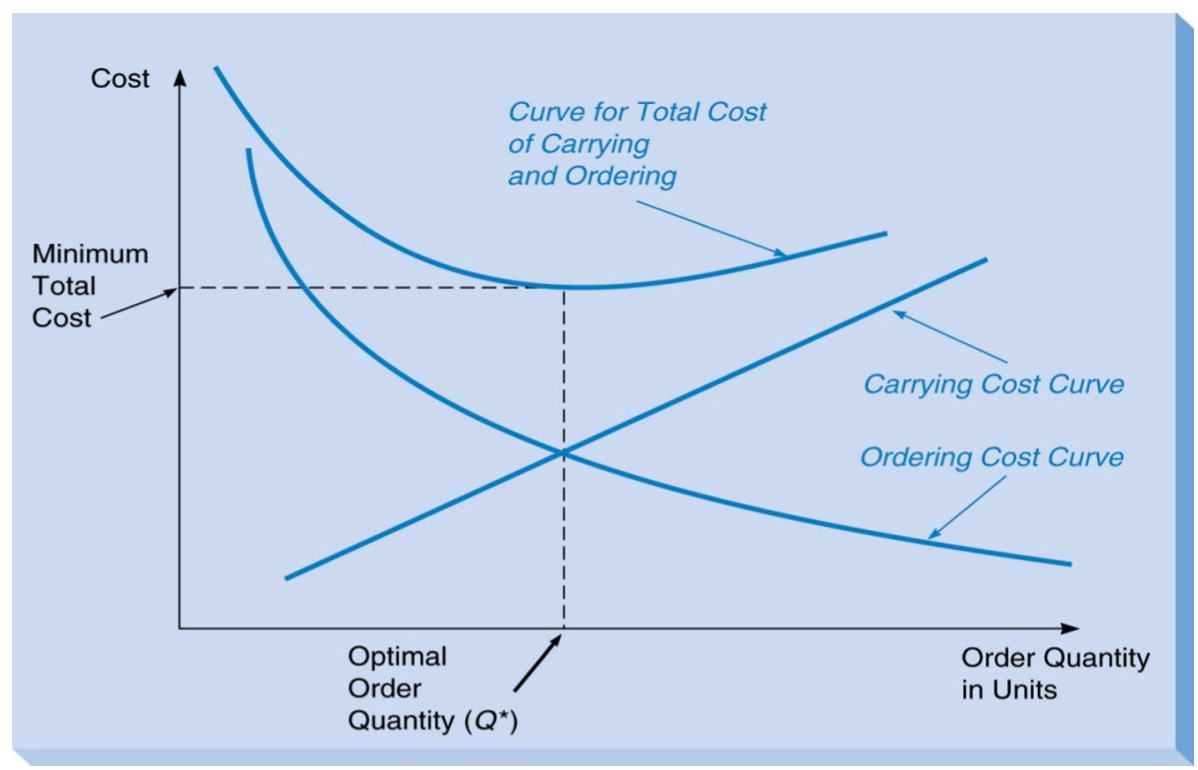

Fig. 1 Optimum EOQ graph

The total annual inventory cost is the sum of the ordering and carrying costs: $T C=\frac{A D}{Q}+\frac{h Q}{2}$.

\section{Some Basic Definition and Results of Matrix}

A matrix is an ordered set of numbers listed rectangular form. If a matrix $A$ has $n$ rows and $n$ columns then we say it's a square matrix. A diagonal matrix is a square matrix with all non-diagonal elements are zero. The diagonal matrix is completely defined by the diagonal elements. When all the elements of a matrix $A$ are zero, we call $A$ as a null matrix. An identity matrix $I$ is a diagonal matrix with all the diagonal elements is equal to one. A matrix function is a function which maps a matrix to another matrix. Let $A$ be a square matrix of order $n$. Then the trace of the matrix is the sum of the main diagonal elements. The trace of a matrix $A$ is also commonly denoted as $\operatorname{Tr}(A)$. Let $A^{-1}$ be the inverse matrix of a matrix $A$, then $A^{-1} A=A A^{-1}=I$. Matrix $B$ is said to be a square root of a matrix $A$ if the matrix product $B B$ is equal to $A$. In general, a matrix can have many square roots. For example, the matrix $\left[\begin{array}{ll}33 & 24 \\ 48 & 57\end{array}\right]$ has square roots $\left[\begin{array}{ll}1 & 4 \\ 8 & 5\end{array}\right]$ and $\left[\begin{array}{ll}5 & 2 \\ 4 & 7\end{array}\right]$, as well as their additive inverses some matrices have no square root. An example is the non square root matrix is $\left[\begin{array}{ll}0 & 1 \\ 0 & 0\end{array}\right]$. If $D$ is a diagonal $n \times n$ matrix, one can obtain a square root by taking a diagonal matrix $R$, where each element along the diagonal is a square root of the corresponding element of $D$. If the diagonal elements of $D$ are real and non-negative, and the square roots are taken with non-negative sign, the matrix $R$ will be the principal root of $D$. For example $\left[\begin{array}{cc}81 & 0 \\ 0 & 9\end{array}\right]$ has a square root $\left[\begin{array}{ll}9 & 0 \\ 0 & 3\end{array}\right]$. 
Eriksen [3] presented the definitions 1-4.

Definition 1 Let $f\left(x_{1}, x_{2}, \ldots, x_{n}\right)$ be a function in $n$ variables. Then $x$ is said to be a stationary point of $f$ if $\frac{\partial f(x)}{\partial x_{1}}=\frac{\partial f(x)}{\partial x_{2}}=\cdots=\frac{\partial f(x)}{\partial x_{n}}=0$.

Definition 2 Let $f(x)$ be a function in $n$ variables. The Hessian matrix of $f$ is the matrix consisting of all the second order partial derivatives of $f$. That is, the Hessian matrix of $f$ at the point $x$ is the $n \times n$ matrix

$$
H=\left[\begin{array}{ccccc}
\frac{\partial^{2} f}{\partial x_{1}^{2}} & \frac{\partial^{2} f}{\partial x_{1} \partial x_{2}} & \cdot & \cdot & \frac{\partial^{2} f}{\partial x_{1} \partial x_{n}} \\
\frac{\partial^{2} f}{\partial x_{2} \partial x_{1}} & \frac{\partial^{2} f}{\partial x_{2}^{2}} & \cdot & \cdot & \frac{\partial^{2} f}{\partial x_{2} \partial x_{n}} \\
\cdot & \cdot & \cdot & \cdot & \cdot \\
\cdot & \cdot & \cdot & \cdot \\
\frac{\partial^{2} f}{\partial x_{n} \partial x_{1}} & \cdot & \cdot & \cdot & \frac{\partial^{2} f}{\partial x_{n}^{2}}
\end{array}\right] .
$$

Definition 3 Let $A$ ba a symmetric $n \times n$ matrix. Then

(i) $A$ is positive definite iff $D_{k}>0$ for all leading principal minors.

(ii) $A$ is negative definite iff $(-1)^{k} D_{k}>0$ for all leading principal minors.

(iii) $A$ is positive semidefinite iff $\Delta_{k} \geq 0$ for all principal minors.

(iv) $A$ is negative semidefinite iff $(-1)^{k} \Delta_{k} \geq 0$ for all principal minors.

Definition 4 Let $f(x)$ be a function in $n$ variables and $H$ be a Hessian matrix of $f$. Then at a point $x$

(i) $f$ is convex $\Leftrightarrow H$ is positive semidefinite.

(ii) $f$ is concave $\Leftrightarrow H$ is negative semidefinite.

(iii) $f$ is strictly convex $\Leftrightarrow H$ is positive definite.

(iv) $A$ is strictly concave $\Leftrightarrow H$ is negative definite.

Theorem 5 If $A=\operatorname{diag}(a, b, c)$, then $A \cdot A=\operatorname{diag}\left(a^{2}, b^{2}, c^{2}\right), A \cdot A \cdot A=$ $\operatorname{diag}\left(a^{3}, b^{3}, c^{3}\right), \ldots$

This property can be generalized for $A=\operatorname{diag}(a, b, c, d, e, \ldots)$.

Theorem 6 Let $A=\left[\begin{array}{cccc}a_{11} & 0 & \cdots & 0 \\ 0 & a_{22} & \cdots & 0 \\ \cdot & \cdot & \cdots & \cdot \\ \cdot & \cdot & \cdots & \cdot \\ \cdot & \cdot & \cdots & a_{n n}\end{array}\right]$ be an $n \times n$ diagonal matrix. Then its inverse is

given by: $A^{-1}=\left[\begin{array}{cccc}\frac{1}{a_{11}} & 0 & \cdots & 0 \\ 0 & \frac{1}{a_{22}} & \cdots & 0 \\ \cdot & \cdot & \cdots & . \\ \cdot & \cdot & \cdots & \cdot \\ \cdot & \cdot & \cdots & \frac{1}{a_{n n}}\end{array}\right]$ provided that none of the diagonal elements are zero. If any of the diagonal elements are zero then $A$ is not invertible.

Proof Consider the right inverse of $A$. 
Case 1 Suppose none of the diagonal elements are zero. Then by the definition of inverse, our assertion is that the matrix product of the two matrices in question is the identity matrix of order $n$.

Now, observe that:

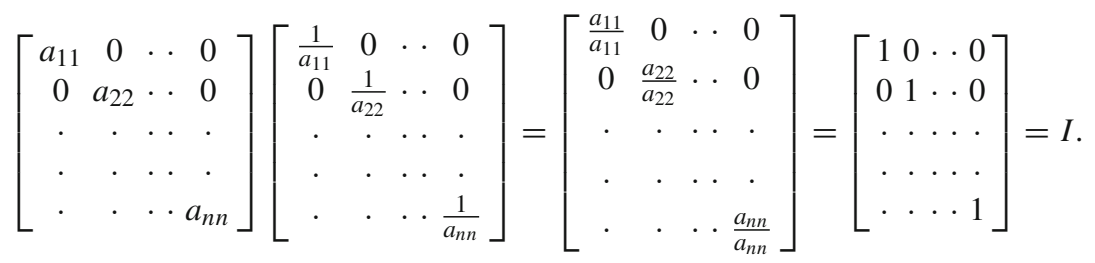

Case 2 suppose one of the diagonal elements is zero. Then $\operatorname{det}(A)=0$, where det indicates the determinant of $A$. From determinant of inverse it would follow that: $\operatorname{det}\left(A^{-1}\right)=1 / \operatorname{det}(A)$. But this equation has no solution, and so $A$ cannot admit an inverse. Hence proved.

\section{Literature Review}

In the classical inventory models, the demand rate is regularly assumed to be either constant or time-dependent but independent of the stock levels. However, practically an increase in shelf space for an item induces more consumers to buy it. This occurs owing to its visibility, popularity or variety. Conversely, low stocks of certain goods might raise the perception that they are not fresh. Therefore, it is observed that the demand rate may be influenced by the stock levels for some certain types of inventory. In years, marketing researchers and practitioners have recognized the phenomenon that the demand for some items could be based on the inventory level on display. Levin et al. [4] pointed out that large piles of consumer goods displayed in a supermarket would attract the customer to buy more. Silver and Peterson [5] noted that sales at the retail level tend to be proportional to stock displayed. Banerjee [6] demonstrated the joint economic lot-size model for purchaser and vendor. Goyal [7] illustrated that manufacturing a batch which is made up of an integral number of equal shipments generally produces a lower cost solution. Baker and Urban [8] established an EOQ model for a power-form inventory-level-dependent demand pattern. Padmanabhan and Vrat [9] developed a multi-item inventory model of deteriorating items with stock-dependent demand under resource constraints and solved by a non-linear goal programming method. Datta and Pal [10] presented an inventory model in which the demand rate is dependent on the instantaneous inventory level until a given inventory level is achieved, after which the demand rate becomes constant. Urban [11] relaxed the unnecessary zero ending-inventory at the end of each order cycle as imposed in Datta and Pal [10]. Pal et al. [12] extended the model of Baker and Urban [8] for perishable products that deteriorate at a constant rate. Bar-Lev et al. [13] developed an extension of the inventory-level-dependent demand-type EOQ model with random yield. Gerchak and Wang [14] constructed the periodic-review inventory models with inventory-level dependent demand. Padmanabhan and Vrat [15] proposed an EOQ models for perishable items under stock dependent selling rate. Ray and Chaudhuri [16] demonstrated an EOQ model with stock-dependent demand, shortage, inflation and time discounting. Roy and Maiti [17] established the multi-objective inventory models of deteriorating items with some constraints in a fuzzy environment. Hwang and Hahn [18] presented an optimal procurement policy for items with an inventory level-dependent demand rate and fixed lifetime. Datta and Paul [19] developed an inventory system with stock-dependent, price-sensitive demand rate. 
Kar et al. [20] described an inventory of multi-deteriorating items sold from two shops under single management with constraints on space and investment. Sana [21], proposed a finite time-horizon deterministic EOQ model where the rate of demand decreases quadratically with selling price. Prices at different periods are considered as decision variables. Sana [22], investigated the retailers optimal order quantity for similar products. It is assumed that the amount of display space is limited and the demand of the products depends on the display stock level where more stock of one product makes a negative impression of another product. Besides it, the demand rate is also dependent on selling price and salesmens initiatives. Also, the replenishment rate depends on the level of stock of the items. Cárdenas-Barrón et al. [23], presented an alternative heuristic algorithm to solve the vendor management inventory system with multi-product and multi-constraint based on EOQ with backorders considering two classical backorders costs as linear and fixed. Sana [24], explained the retailers optimal order quantity for similar products. It is assumed that the amount of display space is limited and the demand of the products depends on the display stock level and the initiatives of sales staff where more stock of one product makes a negative impression of the another product. Also, the replenishment rates depend on the level of stocks of the items. Sana [25], studied empirically develops a framework of an EOQ model for conforming and nonconforming quality products, while the nonconforming items, a random proportionate of the total products, are sold at a reduced price after a $100 \%$ screening process. In general, a trade credit policy is offered to the retailer by the supplier to buy more items. In this point of view, different delay periods with different purchasing costs are settled for the retailer. The associated expected average profit for both conforming and nonconforming products by trading off selling prices, inventory costs, interest earned from selling items, interest charged for delayed payments of purchasing cost, and shortage costs is maximized analytically. Sana [26], presented an EOQ model for uncertain demand when capacity of own warehouse is limited and the rented warehouse is considered, if needed. The expected average cost function is formulated for both continuous and discrete distributions of demand function by trading off holding costs and stock out penalty. Cárdenas-Barrón et al. [27], proposed model to determines both the optimal replenishment lot size and the optimal number of shipments jointly. De and Sana [28], discussed an intuitionistic fuzzy EOQ inventory model with backlogging is investigated using the score functions for the member and nonmembership functions. The demand rate is varying with selling price and promotional effort (PE). A crisp model is formulated first. Then, intuitionistic fuzzy set and score function (or net membership function) are applied in the proposed model, considering selling price and PE as fuzzy numbers. To obtain the best inventory policy, ranking index method has been adopted, showing that the score function can maintain the ranking rule also. Moreover, optimization is made under the general fuzzy optimal and intuitionistic fuzzy optimal policy. Tayal et al. [29], to developed a model for a two echelon supply chain, in which retailers' demand is seasonal with expiration date and he invests on the preservation technology to reduce the rate of product deterioration. A solution procedure is presented to determine an optimal replenishment cycle, shortage period and preservation technology cost such that the net profit for the supply chain is maximized. De et al. [30], described fuzzyfies EOQ crisp model with backlogged while demand of the customers varies with selling price and PE. The demand rate in stock out situation decreases with duration of the shortage period and it comes back to its initial rate since PE continues. The cost function consists of set up cost, inventory cost, shortage cost and cost for PE. The coefficient vectors of the concerned minimization cost function are considered as fuzzy numbers which are transformed into interval numbers. Then, the interval objective function has been transformed into a classical multi-objective EOQ model using intuitionistic fuzzy technique. Tsoularis [31], proposed a 
logistic growth model for the inventory dependent demand rate and solve first the continuous time deterministic optimal control problem of maximising the present value of the total net profit over an infinite horizon. It is shown that under a strict condition there is a unique optimal stock level which the inventory planner should maintain in order to satisfy demand. Pal et al. [32], proposed an imperfect EPQ (economic production quantity) price dependent inventory model over two types of cycles: in the first cycle, the retailer sells only good product with actual price and, in the second, he sells the products with a discount price. In the production run-time, the non-conforming items are produced at a random rate and they are reworked after the regular production run time and the reworked items are almost perfect as good as original quality items. The retailer starts the second cycle when a certain percent of good items are left to him, after the completion of regular production. The retailer continues simultaneously two cycles up to which both types of the products are available to him. Cárdenas-Barrón et al. [33], proposed an alternative heuristic algorithm for a multi-product EPQ vendor-buyer integrated model with just in time philosophy and a budget constraint. Soni and Patel [34], investigated a single-vendor-single-buyer production inventory model involving defective items in both an individual and joint management system with service level constraint. In the system under study, the vendor produces a product in a batch with finite production rate and transfers the lot in equal size to the buyer facing stochastic demand, which is assumed to be normally distributed. The lead time can be reduced by added crashing cost. Moreover, the backorder rate is dependent on the length of lead time through the amount of shortages and the lead time and ordering cost reduction are interacted.

In "Assumptions and Notations are Used in Inventory Models" section, we provide the fundamental assumptions for the proposed EOQ model and the notations used throughout this paper. In "Main Result of the Model" section, we set up a mathematical model. Theorem 7 and Proposition 8 is provided to show the characteristics of the optimal solution. In "Algorithm" section, solution algorithm is developed. In "Numerical Example" section, numerical examples are presented. Finally, in "Conclusion" section, we draw the conclusions and address possible future work.

\section{Assumptions and Notations are Used in Inventory Models}

Assumptions

- Demand is known and uniform.

- Shortages are not allowed.

- The order quantity is received instantaneously.

- Lead time is zero.

- Demand, order size of $n$ products are defined as a diagonal matrix of order $n \times n$.

- Each cost is defined as a diagonal matrix of order $n \times n$ such cost are ordering cost, holding cost, total cost for $n$ products.

\section{Notations}

$i \quad$ Number of products are assumed in the model $(i=1-n)$.

$D_{i} \quad$ total demand (in units) for the $i$ th product.

$D$ diagonal demand matrix of order $n \times n$ and each diagonal elements are denoted the demand of the $i$ th products (in units), respectively.

$Q_{i} \quad$ optimal order quantity (in units) for the $i$ th product. 
$Q \quad$ diagonal ordering size matrix of order $n \times n$ and each diagonal elements are denoted the ordering size of the $i$ th products (in units), respectively.

$A_{i} \quad$ ordering cost (per order) for the $i$ th product.

A diagonal ordering cost matrix of order $n \times n$ and each diagonal elements are denoted the ordering cost of the $i$ th products (in units), respectively.

$h_{i} \quad$ carrying cost per unit carried for the $i$ th product.

$H \quad$ diagonal carrying cost matrix of order $n \times n$ and each diagonal elements are denoted the carrying cost of the $i$ th products (in units), respectively.

$T C_{i} \quad$ minimum total inventory cost for the $i$ th product.

TC diagonal total inventory cost matrix of order $n \times n$ and each diagonal elements are denoted the total inventory cost of the $i$ th products (in units), respectively.

\section{Main Result of the Model}

In this section a theorem and a proposition provided to show the characteristics of the optimal solution.

Theorem 7 Let $D, A, H, T C, Q$ be the $n \times n$ diagonal matrix of demand, ordering cost, holding cost and total inventory cost and order size of $n$ items and each diagonal elements of a matrix are denoted by the respective cost of each items.

Then

(i) Diagonal ordering size matrix of order $n \times n$ is $Q=\sqrt{2 D A H^{-1}}$.

$$
\text { That is, } Q=\left[\begin{array}{cccc}
Q_{1} & 0 & \cdots & 0 \\
0 & Q_{2} & \cdots & 0 \\
\cdot & \cdot & \cdots & \cdot \\
. & . & \cdots & \cdot \\
0 & 0 & \cdots & Q_{n}
\end{array}\right] \text {, where } Q_{i}=\sqrt{\frac{2 D_{i} A_{i}}{h_{i}}} \text {. }
$$

(ii) Diagonal total inventory cost matrix is $T C=D A Q^{-1}+\frac{Q H}{2}$

$$
\text { that } i s, T C=\left[\begin{array}{cccc}
T C_{1} & 0 & \cdots & 0 \\
0 & T C_{2} & \cdots & 0 \\
. & \cdot & . & . \\
. & . & \cdots & . \\
0 & 0 & \cdots & T C_{n}
\end{array}\right] \text { where } T C_{i}=\frac{D_{i} A_{i}}{Q_{i}}+\frac{Q_{i} h_{i}}{2} \text {. }
$$

(iii) Total inventory cost for all products is equal to the trace of the total inventory cost diagonal matrix. That is, total inventory cost for all products $=\operatorname{Tr}(T C)=\sum_{i} T C_{i}$.

(iv) Show that the total inventory cost is a convex function.

Proof Let $D_{1}, D_{2}, \ldots, D_{n}$ are the demand of $n$ products, respectively, Similarly $A_{1}, A_{2}, \ldots$, $A_{\mathrm{n}}, h_{1}, h_{2}, \ldots, h_{n}, T C_{1}, T C_{2}, \ldots, T C_{n}$ and $Q_{1}, Q_{2}, \ldots, Q_{n}$ are the ordering cost, holding cost, total inventory cost and ordering size of $n$ products, respectively. 
Now to define diagonal matrix for demand, ordering cost, holding cost, ordering size and Total inventory cost.

$$
\begin{aligned}
\text { Let } D & =\left[\begin{array}{cccc}
D_{1} & 0 & \cdots & 0 \\
0 & D_{2} & \cdots & 0 \\
\cdot & \cdot & \cdots & \cdot \\
\cdot & \cdot & \cdots & \cdot \\
0 & 0 & \cdots & D_{n}
\end{array}\right], Q=\left[\begin{array}{cccc}
Q_{1} & 0 & \cdots & 0 \\
0 & Q_{2} & \cdots & 0 \\
\cdot & \cdot & \cdots & \cdot \\
\cdot & \cdot & \cdots & \cdot \\
0 & 0 & \cdots & Q_{n}
\end{array}\right], \quad H=\left[\begin{array}{cccc}
h_{1} & 0 & \cdots & 0 \\
0 & h_{2} & \cdots & 0 \\
\cdot & \cdot & \cdots & \cdot \\
\cdot & \cdot & \cdots & \cdot \\
0 & 0 & \cdots & h_{n}
\end{array}\right], \\
A & =\left[\begin{array}{cccc}
A_{1} & 0 & \cdots & 0 \\
0 & A_{2} & \cdots & 0 \\
\cdot & \cdot & \cdots & \cdot \\
\cdot & \cdot & \cdots & \cdot \\
0 & 0 & \cdots & A_{n}
\end{array}\right], \text { and } T C=\left[\begin{array}{cccc}
T C_{1} & 0 & \cdots & 0 \\
0 & T C_{2} & \cdots & 0 \\
\cdot & \cdot & \cdots & \cdot \\
\cdot & \cdot & \cdots & \cdot \\
0 & 0 & \cdots & T C_{n}
\end{array}\right] .
\end{aligned}
$$

(i) In Fig. 1 shows the inverse relationship between ordering cost and carrying cost, resulting in a convex total cost curve. The optimal order quantity occurs at the point in Fig. 1 where the total cost curve is at a minimum, which coincides exactly with the point where the carrying cost curve intersects the ordering cost curve. This enables us to determine the optimal value of $Q$ by equating the two cost matrices and solving for $Q$ :

$$
\begin{gathered}
\text { Hence } \frac{D A}{Q}=\frac{Q H}{2}=>\quad Q^{2}=\frac{2 D A}{H}=2 D A H^{-1} . \\
{\left[\begin{array}{cccc}
Q_{1} & 0 & \cdots & 0 \\
0 & Q_{2} & \cdots & 0 \\
\cdot & \cdot & \cdots & \cdot \\
\cdot & \cdot & \cdots & \cdot \\
0 & 0 & \cdots & Q_{n}
\end{array}\right]=\left[\begin{array}{cccc}
\frac{2 D_{1} A_{1}}{h_{1}} & 0 & \cdots & 0 \\
0 & \frac{2 D_{2} A_{2}}{h_{2}} & \cdots & 0 \\
\cdot & \cdot & \cdots & \cdot \\
\cdot & \cdot & \cdots & \cdot \\
0 & 0 & \cdots & \frac{2 D_{n} A_{n}}{h_{n}}
\end{array}\right],} \\
{\left[\begin{array}{cccc}
Q_{1} & 0 & \cdots & 0 \\
0 & Q_{2} & \cdots & 0 \\
\cdot & \cdot & \cdots & \cdot \\
\cdot & \cdot & \cdots & \cdot \\
0 & 0 & \cdots & Q_{n}
\end{array}\right]=\left[\begin{array}{cccc}
\sqrt{\frac{2 D_{1} A_{1}}{h_{1}}} & 0 & \cdots \\
0 & \sqrt{\frac{2 D_{2} A_{2}}{h_{2}}} & \cdots & 0 \\
\cdot & \cdot & \cdots & \cdot \\
\cdot & \cdot & \cdots & \cdot \\
0 & 0 & \cdots & \sqrt{\frac{2 D_{n} A_{n}}{h_{n}}}
\end{array}\right] .}
\end{gathered}
$$

Therefore $Q=\sqrt{2 D A H^{-1}}$ and $Q_{i}=\sqrt{\frac{2 D_{i} A_{i}}{h_{i}}}$. Hence (i) proved.

(ii) From the definition of total inventory cost we get the following matrix

$$
\begin{aligned}
T C & =\frac{D A}{Q}+\frac{Q H}{2}, \\
T C & =D A Q^{-1}+\frac{Q H}{2} \text { since } Q^{-1} \text { is the inverse matrix of } Q,
\end{aligned}
$$




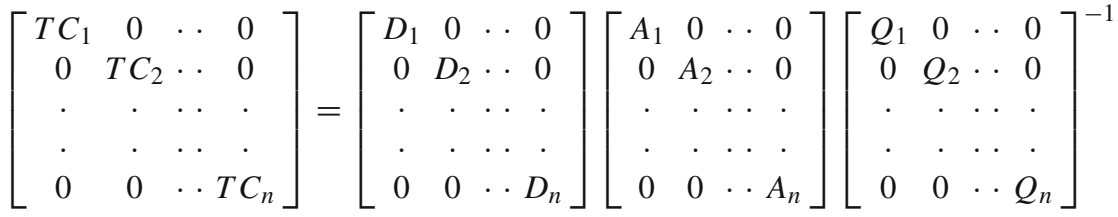

$$
\begin{aligned}
& +\frac{1}{2}\left[\begin{array}{cccc}
Q_{1} & 0 & \cdots & 0 \\
0 & Q_{2} & \cdots & 0 \\
\cdot & \cdot & \cdots & . \\
. & . & \cdots & . \\
0 & 0 & \cdots & Q_{n}
\end{array}\right]\left[\begin{array}{cccc}
h_{1} & 0 & \cdots & 0 \\
0 & h_{2} & \cdots & 0 \\
\cdot & \cdot & \cdots & . \\
\cdot & \cdot & \cdots & . \\
0 & 0 & \cdots & h_{n}
\end{array}\right] \text {, }
\end{aligned}
$$

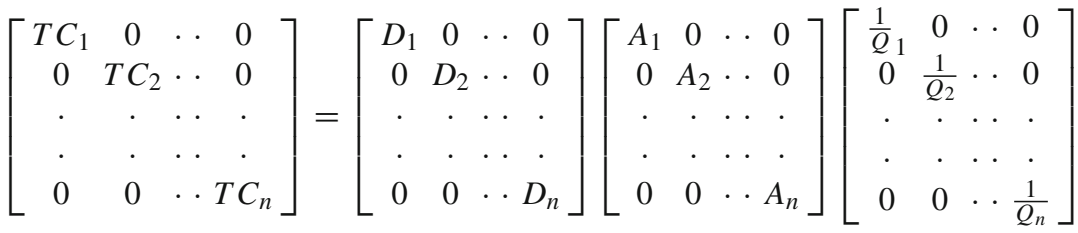$$
+\frac{1}{2}\left[\begin{array}{cccc}
Q_{1} & 0 & \cdots & 0 \\
0 & Q_{2} & \cdots & 0 \\
\cdot & \cdot & \cdots & \cdot \\
\cdot & \cdot & \cdots & \cdot \\
0 & 0 & \cdots & Q_{n}
\end{array}\right]\left[\begin{array}{cccc}
h_{1} & 0 & \cdots & 0 \\
0 & h_{2} & \cdots & 0 \\
\cdot & \cdot & \cdots & \cdot \\
\cdot & \cdot & \cdots & \cdot \\
0 & 0 & \cdots & h_{n}
\end{array}\right] \text {, }
$$

$$
\begin{aligned}
& {\left[\begin{array}{cccc}
T C_{1} & 0 & \cdots & 0 \\
0 & T C_{2} & \cdots & 0 \\
\cdot & \cdot & \cdots & \cdot \\
\cdot & \cdot & \cdots & \cdot \\
0 & 0 & \cdots & T C_{n}
\end{array}\right]=\left[\begin{array}{cccc}
\frac{D_{1} A_{1}}{Q_{1}} & 0 & \cdots & 0 \\
0 & \frac{D_{2} A_{2}}{Q_{2}} & \cdots & 0 \\
\cdot & \cdot & \cdots & \cdot \\
\cdot & \cdot & \cdots & \cdot \\
0 & 0 & \cdots & \frac{D_{n} A_{n}}{Q_{n}}
\end{array}\right]+\left[\begin{array}{cccc}
\frac{Q_{1} h_{1}}{2} & 0 & \cdots & 0 \\
0 & \frac{Q_{2} h_{2}}{2} & \cdots & 0 \\
\cdot & \cdot & \cdots & \cdot \\
\cdot & \cdot & \cdots & \cdot \\
0 & 0 & \cdots & \frac{Q_{n} h_{n}}{2}
\end{array}\right],} \\
& T C=\left[\begin{array}{cccc}
\frac{D_{1} A_{1}}{Q_{1}}+\frac{Q_{1} h_{1}}{2} & 0 & \cdots & 0 \\
0 & \frac{D_{2} A_{2}}{Q_{2}}+\frac{Q_{2} h_{2}}{2} & \cdots & 0 \\
\cdot & \cdot & \cdots & \cdot \\
\cdot & \cdot & \cdots & \cdot \\
0 & 0 & \cdots & \frac{D_{n} A_{n}}{Q_{n}}+\frac{Q_{n} h_{n}}{2}
\end{array}\right], \\
& \therefore T C_{i}=\frac{D_{i} A_{i}}{Q_{i}}+\frac{Q_{i} h_{i}}{2} \text {. }
\end{aligned}
$$

Hence (ii) is proved.

(iii) From (ii) it is obvious that total inventory cost for $n$ items is equal to the trace of the diagonal total inventory cost matrix.

That is, total inventory cost for all $n$ products $=\sum_{i} T C_{i}=\sum_{i}\left(\frac{D_{i} A_{i}}{Q_{i}}+\frac{Q_{i} h_{i}}{2}\right)$. Hence (iii) proved.

(iv) Let $T C(Q)=\sum_{i}\left(\frac{D_{i} A_{i}}{Q_{i}}+\frac{Q_{i} h_{i}}{2}\right)$ be a total inventory cost function of $n$ variables $Q_{1}, Q_{2}, \ldots, Q_{n}$.

To show that $T C(Q)$ be convex it is enough to prove the Hessian matrix of $T C(Q)$ is either positive semidefinite or positive definite.

We have that the first order partial derivatives of $T C(Q)$ with respect to $Q_{1}, Q_{2}, \ldots, Q_{n}$ are $\frac{\partial T C(Q)}{\partial Q_{1}}=-\frac{D_{1} A_{1}}{Q_{1}^{2}}+\frac{h_{1}}{2}, \frac{\partial T C(Q)}{\partial Q_{2}}=-\frac{D_{2} A_{2}}{Q_{2}^{2}}+\frac{h_{2}}{2}, \ldots, \frac{\partial T C(Q)}{\partial Q_{n}}=-\frac{D_{n} A_{n}}{Q_{n}^{2}}+\frac{h_{n}}{2}$. 
This gives the Hessian matrix as

$$
H=\left[\begin{array}{ccccc}
\frac{\partial^{2} T C(Q)}{\partial Q_{1}^{2}} & \frac{\partial^{2} T C(Q)}{\partial Q_{1} \partial Q_{2}} & \cdot & \cdot & \frac{\partial^{2} T C(Q)}{\partial Q_{1} \partial Q_{n}} \\
\frac{\partial^{2} T C(Q)}{\partial Q_{2} \partial Q_{1}} & \frac{\partial^{2} T C(Q)}{\partial Q_{2}^{2}} & \cdot & \cdot & \frac{\partial^{2} T C(Q)}{\partial Q_{2} \partial Q_{n}} \\
\cdot & \cdot & \cdot & \cdot & \cdot \\
\cdot & \cdot & \cdot & \cdot & \cdot \\
\frac{\partial^{2} T C(Q)}{\partial Q_{n} \partial Q_{1}} & \cdot & \cdot & \cdot & \frac{\partial^{2} T C(Q)}{\partial Q_{n}^{2}}
\end{array}\right]=\left[\begin{array}{cccc}
\frac{2 D_{1} A_{1}}{Q_{1}^{3}} & 0 & \cdots & 0 \\
0 & \frac{2 D_{2} A_{2}}{Q_{2}^{3}} & \cdots & 0 \\
\cdot & \cdot & \cdots & . \\
\cdot & \cdot & \cdots & \cdot \\
0 & 0 & \cdots & \frac{2 D_{n} A_{n}}{Q_{n}^{3}}
\end{array}\right] .
$$

Since all leading principal minor determinants of $H$ are positive. Thus, the Hessian matrix $H$ is positive definite. This implies that the optimal solution of an inventory model would be a minimum.

There fore the total inventory cost is a convex function. Hence (iv) is proved.

Proposition 8 Let $D, A$ and $H$ are diagonal matrices with respect to demand, ordering cost and holding cost. Then the total inventory cost is equal to the trace of square root of $2 \mathrm{DAH}$.

\section{Proof}

$$
\begin{aligned}
& \text { Let } D=\left[\begin{array}{cccc}
D_{1} & 0 & \cdots & 0 \\
0 & D_{2} & \cdots & 0 \\
. & . & . & . \\
. & . & \cdots & . \\
0 & 0 & \cdots & D_{n}
\end{array}\right], \quad Q=\left[\begin{array}{cccc}
Q_{1} & 0 & \cdots & 0 \\
0 & Q_{2} & \cdots & 0 \\
. & \cdot & . & . \\
. & . & \cdots & . \\
0 & 0 & \cdots & Q_{n}
\end{array}\right], \quad H=\left[\begin{array}{cccc}
h_{1} & 0 & \cdots & 0 \\
0 & h_{2} & \cdots & 0 \\
. & . & \cdots & . \\
. & . & . & . \\
0 & 0 & \cdots & h_{n}
\end{array}\right] \text {, } \\
& A=\left[\begin{array}{cccc}
A_{1} & 0 & \cdots & 0 \\
0 & A_{2} & \cdots & 0 \\
. & . & \cdots & . \\
. & . & \cdots & . \\
0 & 0 & \cdots & A_{n}
\end{array}\right] \text { and } T C=\left[\begin{array}{cccc}
T C_{1} & 0 & \cdots & 0 \\
0 & T C_{2} & \cdots & 0 \\
. & . & \cdots & . \\
. & . & \cdots & . \\
0 & 0 & \cdots & T C_{n}
\end{array}\right] \text {. }
\end{aligned}
$$

From Theorem 7 (ii) diagonal total inventory cost matrix, $T C=D A Q^{-1}+\frac{Q H}{2}$

$$
\begin{aligned}
& T C=\left[\begin{array}{cccc}
T C_{1} & 0 & \cdots & 0 \\
0 & T C_{2} & \cdots & 0 \\
\cdot & \cdot & \cdots & \cdot \\
. & . & \cdots & \cdot \\
0 & 0 & \cdots & T C_{n}
\end{array}\right]=\left[\begin{array}{cccc}
D_{1} & 0 & \cdots & 0 \\
0 & D_{2} & \cdots & 0 \\
\cdot & \cdot & \cdots & \cdot \\
\cdot & . & \cdots & \cdot \\
0 & 0 & \cdots & D_{n}
\end{array}\right]\left[\begin{array}{cccc}
A_{1} & 0 & \cdots & 0 \\
0 & A_{2} & \cdots & 0 \\
\cdot & \cdot & \cdots & \cdot \\
\cdot & \cdot & \cdots & \cdot \\
0 & 0 & \cdots & A_{n}
\end{array}\right]\left[\begin{array}{cccc}
Q_{1} & 0 & \cdots & 0 \\
0 & Q_{2} & \cdots & 0 \\
\cdot & \cdot & \cdots & \cdot \\
\cdot & \cdot & \cdots & \cdot \\
0 & 0 & \cdots & Q_{n}
\end{array}\right]^{-1} \\
& +\frac{1}{2}\left[\begin{array}{cccc}
Q_{1} & 0 & \cdots & 0 \\
0 & Q_{2} & \cdots & 0 \\
\cdot & \cdot & \cdots & \cdot \\
\cdot & \cdot & \cdots & \cdot \\
0 & 0 & \cdots & Q_{n}
\end{array}\right]\left[\begin{array}{cccc}
h_{1} & 0 & \cdots & 0 \\
0 & h_{2} & \cdots & 0 \\
\cdot & \cdot & \cdots & \cdot \\
\cdot & \cdot & \cdots & \cdot \\
0 & 0 & \cdots & h_{n}
\end{array}\right]
\end{aligned}
$$




$$
\begin{aligned}
T C= & {\left[\begin{array}{cccc}
T C_{1} & 0 & \cdots & 0 \\
0 & T C_{2} & \cdots & 0 \\
\cdot & \cdot & \cdots & \cdot \\
\cdot & \cdot & \cdots & \cdot \\
0 & 0 & \cdots & T C_{n}
\end{array}\right]=\left[\begin{array}{cccc}
D_{1} A_{1} & 0 & \cdots & 0 \\
0 & D_{2} A_{2} & \cdots & 0 \\
\cdot & \cdot & \cdots & \cdot \\
\cdot & \cdot & \cdots & \cdot \\
0 & 0 & \cdots & D_{n} A_{n}
\end{array}\right]\left[\begin{array}{cccc}
\bar{Q}_{1} & 0 & \cdots & 0 \\
0 & \frac{1}{Q_{2}} & \cdots & 0 \\
\cdot & \cdot & \cdots \\
\cdot & \cdot & \cdots & \cdot \\
0 & 0 & \cdots & \frac{1}{Q_{n}}
\end{array}\right] } \\
+ & {\left[\begin{array}{cccc}
\sqrt{\frac{2 D_{1} A_{1}}{h_{1}}} & 0 & \cdots & 0 \\
0 & \sqrt{\frac{2 D_{2} A_{2}}{h_{2}}} & \cdots & 0 \\
\cdot & \cdot & \cdots & \cdot \\
\cdot & \cdot & \cdots & \cdot \\
0 & 0 & \cdots & \sqrt{\frac{2 D_{n} A_{n}}{h_{n}}}
\end{array}\right]\left[\begin{array}{cccc}
h_{1} & 0 & \cdots & 0 \\
0 & h_{2} & \cdots & 0 \\
\cdot & \cdot & \cdots & \cdot \\
\cdot & \cdot & \cdots & \cdot \\
0 & 0 & \cdots & h_{n}
\end{array}\right] . }
\end{aligned}
$$

Since $Q_{i}=\sqrt{\frac{2 D_{i} A_{i}}{h_{i}}}$.

$$
\begin{aligned}
& T C=\left[\begin{array}{cccc}
T C_{1} & 0 & \cdots & 0 \\
0 & T C_{2} & \cdots & 0 \\
\cdot & \cdot & \cdots & \cdot \\
\cdot & \cdot & \cdots & \cdot \\
0 & 0 & \cdots & T C_{n}
\end{array}\right]=\left[\begin{array}{cccc}
D_{1} A_{1} & 0 & \cdots & 0 \\
0 & D_{2} A_{2} & \cdots & 0 \\
\cdot & \cdot & \cdots & \cdot \\
\cdot & \cdot & \cdots & \cdot \\
0 & 0 & \cdots & D_{n} A_{n}
\end{array}\right]\left[\begin{array}{cccc}
\sqrt{\frac{h_{1}}{2 D_{1} A_{1}}} & 0 & \cdots & 0 \\
0 & \sqrt{\frac{h_{2}}{2 D_{2} A_{2}}} & \cdots & 0 \\
\cdot & \cdot & \cdots & \cdot \\
\cdot & \cdot & \cdots & \cdot \\
0 & 0 & \cdots & \sqrt{\frac{h_{n}}{2 D_{n} A_{n}}}
\end{array}\right] \\
& +\frac{1}{2}\left[\begin{array}{cccc}
\sqrt{2 D_{1} A_{1} h_{1}} & 0 & \cdots & 0 \\
0 & \sqrt{2 D_{2} A_{2} h_{2}} & \cdots & 0 \\
\cdot & \cdot & \cdots & \cdot \\
\cdot & \cdot & \cdots & \cdot \\
0 & 0 & \cdots & \sqrt{2 D_{n} A_{n} h_{n}}
\end{array}\right] \text {, } \\
& T C=\left[\begin{array}{cccc}
T C_{1} & 0 & \cdots & 0 \\
0 & T C_{2} & \cdots & 0 \\
\cdot & \cdot & \cdots & \cdot \\
\cdot & \cdot & \cdots & \cdot \\
0 & 0 & \cdots & T C_{n}
\end{array}\right]=\left[\begin{array}{cccc}
\sqrt{\frac{D_{1} A_{1} h_{1}}{2}} & 0 & \cdots & 0 \\
0 & \sqrt{\frac{D_{2} A_{2} h_{2}}{2}} & \cdots & 0 \\
\cdot & \cdot & \cdots & \cdot \\
\cdot & \cdot & \cdots & \cdot \\
0 & 0 & \cdots & \sqrt{\frac{D_{n} A_{n} h_{n}}{2}}
\end{array}\right]
\end{aligned}
$$$$
+\frac{1}{2}\left[\begin{array}{cccc}
\sqrt{2 D_{1} A_{1} h_{1}} & 0 & \cdots & 0 \\
0 & \sqrt{2 D_{2} A_{2} h_{2}} & \cdots & 0 \\
\cdot & \cdot & \cdots & \cdot \\
\cdot & \cdot & \cdots & \cdot \\
0 & 0 & \cdots & \sqrt{2 D_{n} A_{n} h_{n}}
\end{array}\right] \text {, }
$$$$
T C=\left[\begin{array}{cccc}
T C_{1} & 0 & \cdots & 0 \\
0 & T C_{2} & \cdots & 0 \\
\cdot & \cdot & \cdots & \cdot \\
\cdot & \cdot & \cdots & \cdot \\
0 & 0 & \cdots & T C_{n}
\end{array}\right]=\frac{1}{2}\left[\begin{array}{cccc}
\sqrt{2 D_{1} A_{1} h_{1}} & 0 & \cdots & 0 \\
0 & \sqrt{2 D_{2} A_{2} h_{2}} & \cdots & 0 \\
\cdot & \cdot & \cdots & \cdot \\
\cdot & \cdot & \cdots & \cdot \\
0 & 0 & \cdots & \sqrt{2 D_{n} A_{n} h_{n}}
\end{array}\right]
$$$$
+\frac{1}{2}\left[\begin{array}{cccc}
\sqrt{2 D_{1} A_{1} h_{1}} & 0 & \cdots & 0 \\
0 & \sqrt{2 D_{2} A_{2} h_{2}} & \cdots & 0 \\
\cdot & \cdot & \cdots & \cdot \\
\cdot & \cdot & \cdots & \cdot \\
0 & 0 & \cdots & \sqrt{2 D_{n} A_{n} h_{n}}
\end{array}\right],
$$ 
$T C=\left[\begin{array}{cccc}T C_{1} & 0 & \cdots & 0 \\ 0 & T C_{2} & \cdots & 0 \\ \cdot & \cdot & \cdots & \cdot \\ \cdot & \cdot & \cdots & \cdot \\ 0 & 0 & \cdots & T C_{n}\end{array}\right]=\left[\begin{array}{cccc}\sqrt{2 D_{1} A_{1} h_{1}} & 0 & \cdots & 0 \\ 0 & \sqrt{2 D_{2} A_{2} h_{2}} & \cdots & 0 \\ \cdot & \cdot & \cdots & \cdot \\ \cdot & \cdot & \cdots & \cdot \\ 0 & 0 & \cdots & \sqrt{2 D_{n} A_{n} h_{n}}\end{array}\right]=\sqrt{2 D A H}$.

Therefore, the total inventory cost $=\operatorname{Tr}(T C)=\sum_{i} \sqrt{2 D_{i} A_{i} h_{i}}=\operatorname{Tr}(\sqrt{2 D A H})$. Hence proved.

\section{Algorithm}

Step 1 enter the demand, ordering cost and holding cost for all products.

Step 2 define the diagonal matrix based on demand, ordering cost and holding cost for all products.

Step 3 determine ordering quantity diagonal matrix $Q=\sqrt{2 D A H^{-1}}$.

Step 4 determine total inventory cost diagonal matrix $T C=D A Q^{-1}+\frac{Q H}{2}$.

Step 5 determine the total inventory cost is the trace of total inventory cost diagonal matrix (TC).

Step 6 print total inventory cost and size of ordering quantity for each product.

\section{Numerical Example}

Assume a hospital needs three types of medicines from the pharmaceutical company and so the hospital spend the following expenses:

\begin{tabular}{lrlr}
\hline Products & $D_{i}$ & $A_{i}$ & $H_{i}$ \\
\hline 1 & 1,000 & 200 & 10 \\
2 & 1,500 & 150 & 5 \\
3 & 500 & 250 & 25 \\
\hline
\end{tabular}

Solution:

\section{Step 1}

\begin{tabular}{lrlr}
\hline Products & $D_{i}$ & $A_{i}$ & $H_{i}$ \\
\hline 1 & 1,000 & 200 & 10 \\
2 & 1,500 & 150 & 5 \\
3 & 500 & 250 & 25 \\
\hline
\end{tabular}

Step 2 construct diagonal matrices for the given data

$$
D=\left[\begin{array}{ccc}
1,000 & 0 & 0 \\
0 & 1,500 & 0 \\
0 & 0 & 500
\end{array}\right], \quad A=\left[\begin{array}{ccc}
200 & 0 & 0 \\
0 & 150 & 0 \\
0 & 0 & 250
\end{array}\right] \text { and } H=\left[\begin{array}{ccc}
10 & 0 & 0 \\
0 & 5 & 0 \\
0 & 0 & 25
\end{array}\right] \text {. }
$$


Step 3 determine diagonal ordering quantity matrix

$$
Q=\left[\begin{array}{ccc}
200 & 0 & 0 \\
0 & 300 & 0 \\
0 & 0 & 100
\end{array}\right]
$$

Step 4 determine diagonal total inventory cost matrix

$$
T C=\left[\begin{array}{ccc}
2,000 & 0 & 0 \\
0 & 1,500 & 0 \\
0 & 0 & 2,500
\end{array}\right]
$$

Step 5 total inventory cost is 6,000 .

Step 6 print $\rightarrow$ total inventory cost is Rs. 6,000 and size of ordering quantity 200, 300, 100 for the products $\mathrm{A}, \mathrm{B}, \mathrm{C}$, respectively.

\section{Conclusion}

In this paper with the consideration of matrix we proposed an inventory model to obtaining the optimal solution of total inventory cost and size of ordering quantity . This model specially constructed by using a diagonal matrix for dealing multiple items. We presented a theorem and a proposition which describes the optimum result for varies parameters of inventory cost and the convexity of the total inventory cost .With the help of computational algorithm, numerical example is illustrated to justify the model.

For further studies we are planning to extent the mathematical models to consider more factors related to supply chain performance.

Acknowledgments The authors would like to thank anonymous referees for their most valuable, constructive, innovative comments and suggestions that have encouraged the authors to make significant improvements in this paper. This research is supported by the Council of Scientific and Industrial Research, Government of India under the Scheme of CSIR Research Project with CSIR/No. 25(0218)/13/EMR-II/Dated 05.09.2013.

\section{References}

1. Churchman, C.W., Ackoff, R.L., Arnoff, F.L.: Introduction to Operations Research. Wiley, New York (1957)

2. Hadley, G., Whitin, T.M.: Analysis of Inventory Systems. Prentice Hall, Englewood Cliffs (1963)

3. Eriksen, E.: Principal Minors and the Hessian, Lecturer Notes 5. BI Norwegian management, Department of Economics, Norway (2010)

4. Levin, R.I., McLaughlin, C.P., Lamone, R.P., Kottas, J.F.: Productions/Operations Management: Contemporary Policy for Managing Operating Systems. McGraw-Hill, New York (1972)

5. Silver, E.A., Peterson, R.: Decision, Systems for Inventory Management and Production Planning, 2nd edn. Wiley, New York (1985)

6. Banerjee, A.: A joint economic lot-size model for purchaser and vendor. Decis. Sci. 17, 292-311 (1986)

7. Goyal, S.K.: A joint economic-lot-size model for purchaser and vendor: a comment. Decis. Sci. 19, 236-241 (1988)

8. Baker, R.C., Urban, T.L.: A deterministic inventory system with an inventory level dependent demand rate. J. Oper. Res. Soc. 39, 823-831 (1988)

9. Padmanabhan, G., Vrat, P.: Analysis of multi-item inventory systems under resource constraints: a nonlinear goal programming approach. Eng. Costs Prod. Econ. 20, 121-127 (1990)

10. Datta, T.K., Pal, A.K.: A note on an inventory model with inventory level dependent demand rate. J. Oper. Res. Soc. 41, 971-975 (1990) 
11. Urban, T.L.: An inventory model with an inventory-level-dependent demand rate and relaxed terminal conditions. J. Oper. Res. Soc. 43, 721-724 (1992)

12. Pal, S., Goswami, A., Chaudhuri, K.S.: A deterministic inventory model for deteriorating items with stock-dependent rate. Int. J. Prod. Econ. 32, 291-299 (1993)

13. Bar-Lev, S.K., Parlar, M., Perry, D.: On the EOQ model with inventory-level-dependent demand rate and random yield. Oper. Res. Lett. 16, 167-176 (1994)

14. Gerchak, Y., Wang, Y.: Periodic-review inventory models with inventory-level dependent demand. Nav. Res. Logist. 41, 99-116 (1994)

15. Padmanabhan, G., Vrat, P.: EOQ models for perishable items under stock dependent selling rate. Eur. J. Oper. Res. 86, 281-292 (1995)

16. Ray, J., Chaudhuri, K.S.: An EOQ model with stock-dependent demand, shortage, inflation and time discounting. Int. J. Prod. Econ. 53, 171-180 (1997)

17. Roy, T.K., Maiti, M.: Multi-objective inventory models of deteriorating items with some constraints in a fuzzy environment. Comput. Oper. Res. 25, 1085-1095 (1998)

18. Hwang, H., Hahn, K.H.: An optimal procurement policy for items with an inventory level-dependent demand rate and fixed lifetime. Eur. J. Oper. Res. 127, 537-545 (2000)

19. Datta, T.K., Paul, K.: An inventory system with stock-dependent, price-sensitive demand rate. Prod. Plan. Control 12, 13-20 (2001)

20. Kar, S., Bhunia, A.K., Maiti, M.: Inventory of multi-deteriorating items sold from two shops under single management with constraints on space and investment. Comput. Oper. Res. 28, 1203-1221 (2001)

21. Sana, S.S.: Price-sensitive demand for perishable items - an EOQ model. Appl. Math. Comput. 13(217), 6248-6259 (2011)

22. Sana, S.S.: An EOQ model for salesmen's initiatives, stock and price sensitive demand of similar productsA dynamical system, Appl. Math. Comput. 7(218), 3277-3288 (2011)

23. Cárdenas-Barrón, L.E., Treviño-Garza, G., Wee, H.M.: A simple and better algorithm to solve the vendor managed inventory control system of multi-product multi-constraint economic order quantity model. Expert Syst. Appl. 39, 3888-3895 (2012)

24. Sana, S.S.: The EOQ model—a dynamical system. Appl. Math. Comput. 17(218), 8736-8749 (2012)

25. Sana, S.S.: An economic order quantity model for nonconforming quality products. Serv. Sci. 4(4), 331348 (2012)

26. Sana, S.S.: An EOQ model for stochastic demand for limited capacity of own warehouse. Ann. Oper. Res., 1-17 (2013). doi:10.1007/s10479-013-1510-5

27. Cárdenas-Barrón, L.E., Sarkar, B., Treviño-Garza, G.: An improved solution to the replenishment policy for the EMQ model with rework and multiple shipments. Appl. Math. Model. 37, 5549-5554 (2013)

28. De, S.K., Sana, S.S.: Backlogging EOQ model for promotional effort and selling price sensitive demandan intuitionistic fuzzy approach. Ann. Oper. Res. 1-20 (2013). doi:10.1007/s10479-013-1476-3

29. Tayal, S., Singh, S.R., Sharma, R., Chauhan, A.: Two echelon supply chain model for deteriorating items with effective investment in preservation technology. Int. J. Math. Oper. Res. 6, 84-105 (2014)

30. De, S.K., Goswami, A., Sana, S.S.: An interpolating by pass to Pareto optimality in intuitionistic fuzzy technique for a EOQ model with time sensitive backlogging. Appl. Math. Comput. 230, 664-674 (2014)

31. Tsoularis, A.: Deterministic and stochastic optimal inventory control with logistic stock-dependent demand rate. Int. J. Math. Oper. Res. 6, 41-69 (2014)

32. Pal, B., Sana, S.S., Chaudhuri, K.: Joint pricing and ordering policy for two echelon imperfect production inventory model with two cycles. Int. J. Prod. Econ. 155, 229-238 (2014)

33. Cárdenas-Barrón, L.E., Treviño-Garza, G., Widyadana, G.A., Wee, H.-M.: A constrained multi-products EPQ inventory model with discrete delivery order and lot size. Appl. Math. Comput. 230, 359-370 (2014)

34. Soni, H.N., Patel, K.A.: Optimal policies for vendor-buyer inventory system involving defective items with variable lead time and service level constraint. Int. J. Math. Oper. Res. 6, 316-343 (2014) 